\title{
Determination of the $m_{u}$ and $m_{d}$ quark masses from $\eta \rightarrow 3 \pi$ decay
}

\author{
Martin ZDRÁHAL*† \\ Institute of Particle and Nuclear Physics, Faculty of Mathematics and Physics, \\ Charles University in Prague, V Holešovičkách 2, CZ-18000 Prague, Czech Republic \\ E-mail: zdrahaleipnp.troja.mff.cuni.cz
}

Chiral analysis of $\eta \rightarrow 3 \pi$ decay can provide us with information on isospin breaking effects, which is still needed for precise determination of light quark masses using lattice techniques or QCD sum rules. However, this process is affected by large chiral corrections and there are observed discrepancies between the values of Dalitz plot parameters stemming from the standard ChPT computation of its amplitude and those experimentally measured. By using an analytic dispersive representation we have attempted to obtain the information on the masses by taking this discrepancy into account independently of its exact origin. We have performed two distinct analyses of KLOE experimental data on the charged $\eta \rightarrow \pi^{+} \pi^{-} \pi^{0}$ decay using two general sets of assumptions on the physical amplitude and found that both of the sets are compatible with the data and both of them lead to compatible values for $R=\frac{m_{s}-\hat{m}}{m_{d}-m_{u}}$. Our result is $R=37.7 \pm 2.2$. Supplementing this value with the lattice values for $\hat{m}=\left(m_{u}+m_{d}\right) / 2$ and $r=m_{s} / \hat{m}$ from the review by Flavianet Lattice Averaging Group, we obtain the current quark masses of the $u$ and $d$ quarks in $\overline{M S}$ scheme at $\mu=2 \mathrm{GeV}$ equal to $m_{u}=2.23(10) \mathrm{MeV}, m_{d}=4.63(14) \mathrm{MeV}$.

The 2011 Europhysics Conference on High Energy Physics, EPS-HEP 2011,

July 21-27, 2011

Grenoble, Rhône-Alpes, France

\footnotetext{
* Speaker.

${ }^{\dagger}$ This work is supported by the Center for Particle Physics (project no. LC 527) of the Ministry of Education of the Czech Republic. The presented results of the $\eta \rightarrow 3 \pi$ analysis stem from the work done in collaboration with K. Kampf, M. Knecht and J. Novotný.
} 


\section{Introduction}

For a precise determination of the $m_{u}$ and $m_{d}$ quark masses, it is useful to combine isospin symmetric results of lattice or sum-rules QCD techniques with some isospin breaking (IB) study performed in chiral perturbation theory (ChPT) (more details in [1]). A promising process for the later is $\eta \rightarrow 3 \pi$ decay since it proceeds via IB effects and the electromagnetic (EM) contribution to them is strongly suppressed. The amplitude is thus to a good approximation proportional to $\left(m_{u}-m_{d}\right)$ and by comparing its experimentally measured decay rate with its theoretical prediction we have a direct method for determination of this quantity, in our work appearing as $R=\frac{m_{s}-\hat{m}}{m_{d}-m_{u}}$. A computation of this decay amplitude in the framework of ChPT is affected by large chiral corrections - not until the inclusion of chiral two-loop effects [2] the result for $R$ was in reasonable agreement with expectations. However, even then there is still a theoretical challenge to explain satisfactorily the observed discrepancy between the experimentally measured Dalitz plot parameters and their values predicted by [2]. This has inspired various theoretical ${ }^{1}$ studies [3] exploring possible explanations of the discrepancy, namely higher order final state rescatterings, influence of slow convergence of $\pi \pi$ scattering or of $\eta \rightarrow 3 \pi$ amplitude itself, unexpectedly large EM contributions, effects of resonances or with that connected possibility of incorrectly estimated NNLO constants of ChPT $C_{i}$ s. A complication with the existing analyses that try to avoid those problems of ChPT by using alternative approaches is that they do not contain explicitly $m_{q}$, i.e. even if one found a correct energy dependence of the $\eta$ decay by using them, in order to extract any information on $m_{q}$, one unavoidably needs to match it back to ChPT. In the case such approach used different assumptions than ChPT, this matching would bring another sources of errors into the game.

Taking this into account, we have employed in [6] an analytic dispersive parametrization valid to two-loop order. It is constructed using just basic assumptions of quantum field theories together with some observed hierarchy of various contributions to the amplitude and therefore can reproduce very well also the NNLO chiral result. It is of the form $M(s, t, u)=P(s, t, u)+U(s, t, u)$, where $P(s, t, u)$ is a third order polynomial in $s, t, u$ containing six parameters, and the unitarity part $U(s, t, u)$ contains (in the limit $m_{\pi^{ \pm}}=m_{\pi^{0}}$ ) four of these parameters together with additional parameters describing $\pi \pi$ scattering (held fixed). The whole parametrization is given in terms of an analytic expression and all the parameters specific for this $\eta$ decay appear linearly there. Using this parametrization and the data for $\eta \rightarrow \pi^{+} \pi^{-} \pi^{0}$ from [5], we performed the following analyses.

\section{Analysis I: Correcting ChPT $\eta \rightarrow 3 \pi$ result}

By a comparison of the values of Dalitz parameters from the experiment with their predictions from NNLO ChPT, we have found that despite the discrepancy for the individual parameters, they fulfill two relations which are valid in the case the imaginary part of the chiral amplitude is determined correctly [a further relation verifying this would be the value of the neutral parameter $\beta$ (not measured yet)]. It means that the data indicate the possibility that all the difference between the measured amplitude and the one stemming from the ChPT computation can be described by a small real polynomial, i.e. it can also be included into the values of $C_{i}$ s. This inspired our first analysis,

\footnotetext{
${ }^{1}$ On the experimental side there is substantial activity as well (cf. reports of KLOE and WASA@COSY in [4]), as currently there exists only one precise analysis [5] on at least 4 Dalitz parameters of the charged decay $\eta \rightarrow \pi^{+} \pi^{-} \pi^{0}$.
} 
where we have added such polynomial to our parametrization of the ChPT amplitude and fitted it to our KLOE-like distribution. From this one could extract constraints on such phenomenological values of $C_{i}$ s and in addition we have obtained the corrected value of $R=37.7 \pm 2.9$, where the error is estimated from the (slow) convergence of chiral expansion in the first three leading orders.

\section{Analysis II: Direct fit to experimental $\eta \rightarrow 3 \pi$ data}

Our second analysis gave up on explaining the exact origin of the Dalitz parameters discrepancy and assumed instead that independently on the way one would obtain the correct amplitude from QFT computations, it would fulfill the general properties we have employed in the construction of our parametrization, i.e. it will be reproduced well by the parametrization. We have therefore fitted the experimental data with the parametrization. In order to obtain information on $m_{q}$, we need to fix the normalization of the amplitude by matching to ChPT. Thanks to the fact we have analytic expressions, it is sufficient to find just one matching point, i.e. the point where the chiral expansion of the amplitude converges fast. However, since there is no theoretical argument that would choose one such point, we have assumed that if it exists, it will not be an isolated point but a region, where the behaviors of the NNLO chiral amplitude and of the one fitted from data would be similar and also various chiral orders behave in that region well. We have found a prescription for such matching and obtained $R=37.8 \pm 3.3$, where the error is dominated by the error of our fit to [5].

\section{Conclusions}

We have used two analyses based on different assumptions, which can be however fulfilled simultaneously by the genuine physical amplitude. Since both of the analyses worked well and led to the compatible values for $R$, we expect that the assumptions of both of them are met and the determined value of $R$ is correct. Nevertheless, it will be very important in the forthcoming experimental measurements of $\eta \rightarrow \pi^{+} \pi^{-} \pi^{0}$ to verify them since the current analyses are based just on one experiment [5]. In [1] we have shown how one can combine this determination with the isospin symmetric studies on lattice and those using sum-rules techniques in order to obtain the individual quark masses. For instance, by using FLAG averages [7] we obtain

$$
m_{u}(\overline{M S}, \mu=2 \mathrm{GeV})=2.23(10) \mathrm{MeV}, \quad m_{d}(\overline{M S}, \mu=2 \mathrm{GeV})=4.63(14) \mathrm{MeV} .
$$

\section{References}

[1] M. Zdráhal, Nucl. Phys. Proc. Suppl. 219-220 (2011) 68.

[2] J. Bijnens and K. Ghorbani, JHEP 0711 (2007) 030.

[3] G. Colangelo, S. Lanz, E. Passemar, PoS CD09(2009) 047; S. P. Schneider, B. Kubis, C. Ditsche, JHEP 1102 (2011) 028; A. Nehme, arXiv:1106.3491; M. Kolesár, Nucl.Phys.Proc.Suppl. 219-220(2011) 292.

[4] Proceedings of the International PrimeNet Workshop 2011 in Jülich that will appear on arXiv soon.

[5] KLOE Collaboration, F. Ambrosino et al., JHEP 0805 (2008) 006.

[6] K. Kampf, M. Knecht, J. Novotný, M. Zdráhal, Phys. Rev. D 84 (2011) 114015.

[7] G. Colangelo, S. Durr, A. Juttner et al., Eur. Phys. J. C 71 (2011) 1695. 